\title{
Computing Scalar Implicatures
}

\author{
Lyn Frazier \\ University of Massachusetts, Amherst
}

\section{Introduction}

According to the classical Gricean approach (Herbert Paul Grice 1989), if a speaker utters a sentence such as (1) instead of a (relevant) stronger scalemate sentence such as (2), then the listener can infer that the speaker didn't have evidence for (2).

\section{(1) Lucy has two parrots. \\ (2) Lucy has three parrots.}

Various alternatives to this approach have emerged. In particular, Gennaro Chierchia (2004) argued that scalar implicatures are computed in tandem with the semantic computation, but are canceled in Downward Entailing (DE) contexts (or whatever context licenses NPIs). On this approach, scalar implicature computation is closely connected to the grammatical system, not part of a general reasoning system.

The present paper reports the results of a project the larger goal of which is to develop a theory of adult language comprehension, including the principles determining how, when, and why scalar implicatures are computed. The issues that will be addressed revolve around the issue of whether some pragmatic processing is automatic, taking place hand-in-hand with the semantic composition. An issue lurking in the background is the relation between strengthening as in (1) and domain widening for an NPI such as ever, since both may involve (quasi-) grammatical mechanisms concerned with strengthening an utterance relative to competitor utterances. Chierchia's approach also highlights questions concerning the nature of the contexts in which implicatures tend (not) to be drawn. Are DE contexts important (contexts licensing inferences from sets to subsets)? Are Non-veridical contexts important for implicature computation since like DE contexts they may license NPIs (see Anastasia Giannakidou 1998, for example)?

This work was supported by Grant HD-18708 to the University of Massachusetts. I want to thank the audience at SALT 18, Angelika Kratzer and the semantics graduate students at U-Mass for stimulating discussion. In particular, I am indebted to Chris Potts and Charles Clifton for insightful discussion of the material presented here. 
The issue of the role of focus in driving implicature computation will also be addressed. Focus activates alternatives (focus values) which, if organized from stronger to weaker, form the scale assumed to drive scalar implicatures. From psycholinguistic studies, we also know that focus induces deeper processing of constituents by determining the allocation of attentional resources. So, on the face of it, focus should be expected to be central to any account of implicature processing.

Finally, the issue of embedded vs. global implicatures will be addressed. Are local implicatures less likely to be computed than global ones? Although they will not be in the foreground of the present discussion, the paper will also touch on the issue of how scales are set up and activated, and issues arising due to the epistemic states of discourse participants, e.g., whether there are knowlegeable speakers, and expert interlocutors.

There is a growing psycholinguistic literature on implicature processing in adults. Two positions have received particular attention. Relevance Theory (Dan Sperber and Deirdre Wilson 1986) claims that implicatures are costly to compute and are only computed when they are relevant to context. The idea is that implicatures are computed when the gain in discourse coherence is sufficient to offset the cognitive cost of computing the implicature (see Richard Breheny, Napoleon Katsos and John Williams 2006 for one study that has been taken as evidence for the position.) Another approach is the claim that generalized implicatures exist (e.g., Stephen Levinson 2000, also Lawrence Horn 1972, 1989, 1992). On this view, certain implicatures are computed automatically, though they may be canceled in contexts conflicting with the implicature (see Anne Bezuidenhout and James Cooper Cutting 2002, Daniel Grodner et al. 2008; also Lewis Bott and Ira Noveck 2004, Yi Ting Huang and Jesse Snedecker in progress, Ira Noveck and Andres Posada 2003, Ira Noveck et al. 2007.) The existing adult processing research will not be discussed in detail here, since it involves investigations of non-DE contexts, with the exception of Anna Szabolcsi, Lewis Bott and Brian McElree (ms.), who found that an NPI in a DE-context didn't facilitate scalar inferences.

In what follows, the results of several experiments will be described. All of the experiments were conducted together with Chuck Clifton. They were inspired by the proposal of Gennaro Chierchia (2004), and the first few were conducted together with him. Before turning to the issue of implicatures per se, it should be noted that behind Chierchia's approach to implicatures is the idea that there is a natural language deductive system. In a surprising way, this dovetails with the work of Bart Geurts and Frans van der Silk (2005), who have argued that monotonicity may play a special role in interpretation and inference due to the simplicity of inferences involving simple substitution of a more or less specific term, e.g., substitution of a subset for a set. Based on the accuracy with which participants evaluated arguments involving multiple quantifiers, they argued that upward monotonic quantifiers and arguments involving harmonic rather than 
mixed quantifiers (both upward or both downward entailing quantifiers) are less complex than downward entailing quantifiers and arguments involving mixed quantifiers (one upward and one downward entailing).

\section{Montonicity and implicatures}

Is it true that listeners and readers are less likely to draw scalar implicatures in DE contexts than in non-DE contexts? To address this question, a one-item test was administered to roughly two hundred students at the end of an exam. They were given (3a) or (3b) and asked to complete the example by filling in the blank with the first continuation that came to mind, and then to circle a paraphrase of the critical phrase in the fragment corresponding to their interpretation.
a. DE:
If John has two cars, exactly two 2 or more

b. Non-DE: John has two cars. exactly two 2 or more

The strengthened (exactly two) interpretation was chosen 97/109 in the non-DE context but chosen significantly less often, 68/107 times, for the DE context (Chisquare comparing (3a) to $(3 \mathrm{~b})=17.99, \mathrm{p}<.001)$. Thus, although the strengthened interpretation of two cars predominated in both contexts, the DE context did significantly reduce the amount of strengthening.

In a written paraphrase selection study, Gennaro Chierchia, Lyn Frazier and Charles Clifton (submitted) investigated the interpretation of the sentences in (4) to determine whether there was more strengthening of or to an exclusive interpretation in non-DE contexts than in DE contexts. The non-DE contexts are illustrated in (4a) and (4b), where or occurs in the predicate of an affirmative main clause. For the DE-contexts or was placed in the antecedent of a conditional (4c) or in the first argument of every, as in (4d).

a. Jeremy is a child or foreign. He must fill out a form.

(Non-DE: Simple assertion- 67\% Exclusive or)

b. If someone must fill out a form, he is a child or foreign.

(Non-DE: Consequent of conditional- 68\% Exclusive or)

c. If Jeremy is a child or foreign, he must fill out a form.

(DE: Antecedent of conditional- 59\% Exclusive or)

d. Everyone who is a child or foreign must fill out a form.

(DE: Universal- 42\% Exclusive or)

Is the writer talking about someone.... 
who is either a child or foreign, but not both who is a child or foreign or possibly both

As expected, there were fewer strengthened (exclusive or) interpretations in the DE contexts than in the non-DE contexts $(4 \mathrm{a}>4 \mathrm{c}: \mathrm{t}(47)=2.35, \mathrm{p}<.02 ; 4 \mathrm{~b}>4 \mathrm{c}$ : $\mathrm{t}(47)=2.26, \mathrm{p}<.03)$. The universal example also received fewer strengthened interpretations than the conditional $(4 \mathrm{c}>4 \mathrm{~d}: \mathrm{t}(47)=5.05, \mathrm{p}<.001)$.

The difference between if- and every-sentences was not predicted. We speculated that non-accidental and many-time conditionals may involve less strengthening than accidental and one-time conditionals. Attempts to test this experimentally produced mixed results, with only hints that the hypothesis was correct (see Appendix A). So to determine whether there is a genuine difference between if and every, we tested examples where the two examples were near paraphrases of each other, as in (5) in a written paraphrase selection task.

a. The teacher uses handouts or power point. He will satisfy the new regulations.

(53\% Exclusive or )

b. If a teacher uses handouts or power point, he will satisfy the new regulations.

\section{(29\% Exclusive or $)$}

c. Every teacher who uses handouts or power point will satisfy the new regulations.

(31\% Exclusive or )

For these examples, there was no difference in the amount of strengthening observed for the two DE structures $(5 b, c)$. Further, as expected, both $(5 b)$ and (5c) showed less strengthening than the non-DE context (5a). This indicates that the difference in examples like those in (5) was not a necessary or inherent difference between the two DE contexts.

In sum, the present results show that fewer implicatures are drawn in DE contexts than in non-DE contexts. See Danielle Panizza and Gennaro Chierchia (in progress) for an eye movement study of Italian sentences showing effects of implicatures during the reading of DE and non-DE sentences.

\section{DE contexts or any context that licenses NPIs: (Non-) Veridicality}

DE contexts license NPIs such as any. Non-veridical contexts also seem to license any (Anastasia Giannakidou 1998). The question arises, then, as to whether fewer implicatures are computed in non-veridical contexts than in veridical contexts. In ERP studies of German, Heiner Drenhaus, Stephen Frisch and Doug Saddy (2005) found intermediate effects for non-veridical contexts, 
specifically for questions relative to negative or affirmative declaratives, when they investigated the effects of unlicensed polarity items.

In a written paraphrase selection study with Charles Clifton, sentences with or without would in the complement were tested, as in (6). Often the matrix verb varied as well (6).

(6) a. Jason imagined that Sue would go to Paris or Rome.

b. Jason said that Sue went to Paris or Rome.

The non-veridical sentences received $50 \%$ exclusive or responses whereas the neridical sentences received slightly more (55\% exclusive or). However, the difference was thoroughly non-significant. The result is surprising given that all of the non-veridical examples used in the experiment clearly license any and the veridical examples don't. The results suggest that there may be a difference between the contexts that license NPIs and the contexts that block implicatures (though clearly more than a single study is needed to draw any firm conclusions on this matter).

\section{Focus strengthening hypothesis}

Focusing a scalar implicature trigger results in more implicatures being computed because computing the focus value of the trigger requires activating alternatives to the trigger. In an auditory paraphrase selection study (Lyn Frazier, Charles Clifton and Florian Schwarz in progress), the examples in (7) were recorded with a prominent pitch accent on or, or a prominent pitch accent on the auxiliary.

a. Mary will invite Fred OR Sam to the barbecue.

b. Mary WILL invite Fred or Sam to the barbecue.

She will invite Fred or Sam or possibly both; She will invite Fred or Sam but not both.

Forty-eight undergraduate participants listened to the sentences ( 24 were given each list) and selected a paraphrase corresponding to the interpretation they assigned to the sentence by pulling a trigger under the selected paraphrase. The results of the study are presented in Table 1.

Table 1: Proportion of inclusive (and exclusive) choices, and response time (ms)

\begin{tabular}{lcccc}
\hline & Prop inclusive (exclusive) & RT, inclusive & RT, exclusive \\
PA on or & .165 & $(.835)$ & 4048 & 2942 \\
PA on auxiliary & .286 & $(.714)$ & 4322 & 3378
\end{tabular}


The frequency of inclusive (non-strengthened) interpretations was significantly reduced by the pitch accent on or $(\mathrm{F} 1(1,47)=11.59, \mathrm{p}<.001 ; \mathrm{F} 2(1,15)=22.67$, $\mathrm{p}$ $<.001)$. As expected, focus did play a role in triggering implicatures, presumably by invoking alternatives. (See also Arjen Zondervaan this volume).

\section{DE context not confounded with focus}

The DE contexts in the studies described in Section 2 above included conditionals and the first argument of every. Is it possible that the results of those studies suggesting that fewer implicatures are drawn in DE contexts than in non-DE contexts are actually due not to the monotonicity properties of the contexts but instead due to the fact that the implicature trigger is in a position where informational focus is not expected? The extended focus strengthening hypothesis (below) might provide an alternative account of the results presented on DE contexts.

Extended focus strengthening hypothesis: Listeners/readers allocate more attention to material in a focused position than material in an unfocused position (Anne Cutler and Donald J. Foss 1977, Anne Cutler and Jerry Fodor 1979, Paul Hornby 1974, Anthony Sanford and Patrick Sturt 2002), resulting in deeper processing of the material in the focused position. Deeper processing might lead to consideration of alternatives and activation of scales.

In numerous studies of ellipsis, we have found for example that the choice of an inner antecedent for sluicing depends not only on what constituent bears a $\mathrm{L}+\mathrm{H}^{*}$ pitch accent but also on the expected location of informational focus, cf. Lyn Frazier and Charles Clifton 1998, Katy Carlson, Michael Walsh Dickey, Lyn Frazier and Charles Clifton 2008. Therefore, perhaps in the DE-context studies described above readers are responding to expectations about where informational focus is expected.

To insure that focus alone is not responsible for regulating the computation of scalar implicatures, a visual interpretation study was conducted (Lyn Frazier, Charles Clifton and Florian Schwarz in progress). Sentences containing disjunctions were tested in affirmative (8a) and negative (8b) forms. Affirmative sentences were followed by only two paraphrases, as indicated below (8). By contrast, the paraphrases for the negative sentences included the local implicature paraphrase (exclusive or under the scope of negation) in addition to the inclusive and exclusive paraphrases.

a. Maria asked Bob to invite Fred or Sam to the barbecue.

b. Maria asked Bob not to invite Fred or Sam to the barbecue.

2-choice answer for affirmative; 3-choice for negative.

What did Maria ask Bob to do?

To invite Fred or Sam, To invite Fred or Sam, 
but not both

1

To either not invite

Fred or not invite Sam.

1

'Exclusive' possibly both.

2

To not invite Fred To invite neither or both and not invite Sam. but not just one.

2

'Inclusive'
3

'Logical/local'

Table 2 presents the results in terms of proportion of answer 1 choices, as well as the raw data.

Table 2. Frequencies of 1, 2, and 3 answers:

$\begin{array}{lll}1 & 2 & 3\end{array}$

(9a): Proportion $1=0.653$ :

$374 \quad 190 \quad 4$ (these 4 are just errors)

(9b): Proportion $1=0.071$ :

$39494 \quad 31$ (local implicature answer

chosen as often as 'exclusive' answer)

As expected, there was a large and significant drop in the choice of the exclusive (answer 1) interpretation for the negative examples. Presumably expectations about information structure do not differ across the examples and thus the results suggest that focus/information structure alone will not suffice to characterize when scalar implicatures tend (not) to be drawn.

So far, evidence has been presented showing that implicatures are drawn less often in DE than in non-DE contexts. Implicatures are drawn more often when the implicature trigger is focused than when it is not. However, as the last study shows, effects of DE contexts are not due to the implicature trigger being non-focused (or in syntactic positions where focus is not expected, such as antecedents of conditionals or the first argument of every).

We turn next to the question of whether scalar implicatures are drawn online, during the processing of a sentence. To linguists, perhaps it is not clear why one should care whether an implicature is drawn during the comprehension of a sentence, or at some other point in time. However, on a Chierchia sort of approach, where some pragmatic processing goes hand-in-hand with the semantic computation, effects of implicatures are expected even in the absence of deliberative post-sentence judgment tasks, or verification tasks. On the Chierchia view, computing a scalar implicature is part of interpreting the sentence and thus should not be evidenced only in reasoning tasks or with considered judgments.

On a Relevance theory approach, by contrast, implicatures are expected to be drawn only when the cost of drawing them is offset by the gain in contextual coherence. By contrast with the predictions of Relevance theory, the evidence presented in the present paper suggests that even without specific contextual relevance, implicatures tend to get drawn in non-DE contexts. But one might argue that this is due to the nature of the off-line judgment tasks used. Paraphrase 
selection may itself encourage readers or listeners to consider the various interpretations of the sentence even if they wouldn't have during a simple comprehension task without the paraphrase selection task.

A study where participants simply read sentences for comprehension could address this issue. To this end, a self-paced reading experiment was conducted (Lyn Frazier, Charles Clifton and Florian Schwarz in progress). The or sentences in affirmative/negative contexts tested in the paraphrase selection task (8) were followed by either a continuation strongly favoring an exclusive reading or a neutral sentence (which was neutral or weakly biased to the inclusive). Assuming readers tend to draw implicatures in non-DE contexts but tend not to draw them in DE contexts, an interaction was predicted with advantage for Continuation 1 (relative to Continuation 2) greater in the affirmatives than in the negatives. Caution: The continuation sentences differ from each other in multiple ways that might influence their reading times, so it is only the size of the difference between the two continuations in the affirmative context vs. in the negative context that can be interpreted. Examples are given in (9).

a. Maria asked Bob to invite Fred or Sam to the barbecue. She didn't have enough room to invite both. (Strongly biased to exclusive)

b. Maria asked Bob to invite Fred or Sam to the barbecue. It's the first time she's ever cooked outside. (Neutral/Weak bias to inclusive )

c. Maria asked Bob not to invite Fred or Sam to the barbecue. She didn't have enough room to invite both. (Strongly biased to exclusive)

d. Maria asked Bob not to invite Fred or Sam to the barbecue. It's the first time she's ever cooked outside. (Neutral/Weak bias to inclusive)

The results are presented in Table 3. As expected the difference in reading time between the two continuations was larger for the affirmative sentences than for the negative sentences.

Table 3. Reading time in ms. for Sentence 2

Aff Continuation1: 1696-Contin. 1 is fast relative to Contin. 2 (-209 ms.)

Aff Continuation 2: 1905

Neg Continuation1: 1767-Contin. 1 is not fast relative to Contin. 2 (-39 ms.)

Neg Continuation 2: 1806

The mean reading times of Sentence 2 are of particular interest. Continuation 2 was read more slowly than Continuation $1(\mathrm{~F} 1(1,47)=6.4, \mathrm{p}<.02$, but this difference has no clear interpretation because of the lexical differences between the continuations. Of more interest, the interaction between sentence version and continuation was marginally significant $(\mathrm{F} 1(1,47)=3.22, \mathrm{p}<.08$. Given the 
marginality of the critical interaction, we take the result to be interesting and suggestive, but certainly not conclusive. (There were differences among the Continuation 2 items. In addition, the effect seemed to be sensitive to the particular cutoff used for long reading times. Therefore, we are currently pursuing this finding with more tightly controlled materials.) Nevertheless, the results do point in the direction of scalar implicatures being drawn during a simple reading task, without any need for a deliberative judgment.

In a self-paced reading study, Gennaro Chierchia, Lyn Frazier and Charles Clifton (submitted) tested conditional and universal sentences followed by continuations biased to a non-strengthened (two or more) interpretation, as in $(10 \mathrm{a}, \mathrm{c}, 11 \mathrm{a}, \mathrm{c})$ or a strengthened (exactly two) interpretation of cardinals, as in (10bd, 11b,d).

(10) a. If John has two cars,/then I guess that he must be quite rich.

b. If John has two cars, /then the VW outside must belong to someone else.(from Stephen Levinson, 2000)

c. John has two cars./I guess that he must be quite rich.

d. John has two cars. The VW outside must belong to someone else.

(11) a. Every girl who has three brothers/is a tomboy.

b. Every girl who has three brothers/dreads having four brothers.

c. The girl has three brothers./She is a tomboy.

d. The girl has three brothers./She dreads having a fourth one.

Table 4: Mean reading times (ms), second presentation region when context permitted/ or forced a strengthened interpreation.

Conditional Items

( $\mathrm{N}$ or more) (Exactly $\mathrm{N}) \quad$ Difference

Conditional/Quantified $\quad 1839$ (a) $\quad 2370$ (b) 531

Simple $\quad 1764(\mathrm{c}) \quad 2146(\mathrm{~d}) \quad 382$

Quantified Items

Conditional/Quantified $\quad 1476$ (a) $\quad 1865$ (b) $\quad 389$

$\begin{array}{llll}\text { Simple } & 1767(\mathrm{c}) & 1916(\mathrm{~d}) & 149\end{array}$

All Items

Conditional/Quantified $\quad 1658$ (a) 2118 (b) $\quad 460$

Simple $\quad 1766(c) \quad 2030(d) \quad 264$

The results of the experiment revealed two effects. Sentences disambiguated to their strengthened interpretation took longer to read than their counterparts disambiguated to their basic meaning. This finding cannot be interpreted since different material was read in the two cases. The second finding was the interaction of $\mathrm{DE}$ vs. non-DE context by disambiguation type. The interaction 
between simple (non-DE) and Conditional/Quantified (DE) and continuation was significant $(\mathrm{F} 1(1,47)=5.73, \mathrm{p}<02 ; \mathrm{F} 2(1,21)=4.61, \mathrm{p}<.04)$. As predicted, the disambiguation toward the strengthened interpretation increased reading times more for the DE context sentences than the non-DE sentences.

The results of the self-paced reading studies suggest that differential strengthening effects for DE vs. non-DE contexts can be seen on-line.

\section{Embedded implicatures}

\subsection{Embedded versus local implicatures}

Classic Gricean reasoning produces global implicatures, involving the negation of competitors to entire utterances, as in (12b). Global implicatures are weaker than local implicatures, which result from negation of competitors to proper subparts of an utterance, as in (12a).

(12) George believes some of his advisors are crooks. (Gennaro Chierchia, 2004, Benjamin Russell, 2006)

a. George believes not all of his advisors are crooks.

(Local/Embedded implicature)

b. It's not the case that George believes all of his advisors are crooks.

(Global implicature)

Nausiccaa Pouscoulous and Bart Geurts (2007) investigated whether perceivers compute embedded implicatures in three experiments, two in French and one in Dutch. In their first experiment (see Appendix B for a description of the others), participants saw the sentences in (13). Participants had to indicate whether or not they would endorse the inference provided. On a localist view, where embedded implicatures are regularly computed, all of them should go through. On a classic Gricean view, only (13a) should.

(13) a. Jack read some of the Harry Potter books $=>$ He didn't read all of them. (Control)

b. All of the students read some of the Harry Potter books. $=>$ None of them read them all.

c. Jack has to read some of the Harry Potter books. $=>$ He isn't allowed to read them all.

d. Jill thinks that Jack read some of the Harry Potter books. $=>$ She thinks he didn't read them all. 
The results revealed an endorsement rate of $93 \%$ for the control, $27 \%$ for all, $3 \%$ for $m u s t, 50 \%$ for think. As expected by the investigators, the endorsement rate dropped dramatically for the embedded implicatures in (13b-d).

To determine whether there would be a comparable drop in the number of implicatures in embedded contexts in English, I conducted a small pilot study testing two lexicalizations of each of the structures in (13) in a written paraphrase selection study. Based on my own intuitions, I expected more embedded implicatures in English than Pouscoulous and Geurts had found. Further, the pilot was designed to examine whether any drop in the implicatures drawn in embedded contexts might be related to implicit prosody. Greater prominence might be placed on some in the control (13a) than in the embedded examples (13b-d). (Alternatively, the probability of placing a prominent accent early in the sentence, before the direct object, might be greater in (13b-d) than in (13a)).

15 participants were tested. The experimental sentences were divided into three lists. On one, none of the experimental items had a capitalized word, though some of the fillers did. On the other two lists, each sentence appeared once and it contained a capitalized word; the capitalized word was either some or a word earlier in the sentence (all, has, thinks, or, in (13a), Jack), counterbalanced across lists so that half the experimental sentences a participant saw had SOME and half had some. Participants were instructed that capitalized words would be spoken with stress. The materials are illustrated in (14) and (15).

JACK read some of the Harry Potter books. (JACK/SOME) Jack read some but not all of the HP books

Jack read at least some of the HP books

(15) All of the students wrote SOME of the official memos. (ALL/SOME) All of the students wrote some but not all of the official memos All of the students wrote at least some of the official memos

The results of the pilot study are presented in Table 5, where the lefthand column summarizes the Nausicaa Pouscoulous and Bart Geurts results, the middle column reports the sum for all items in the pilot study, and the rightmost column reports the results for just List 1 where no experimental item was capitalized.

Table 5. Percent strengthened interpretations in the pilot study.

$\begin{array}{llll} & \text { P\&G } & \text { Pilot } & \text { Pilot-List 1(=no stress) } \\ \text { Control } & 93 \% & 76 \% & 90 \% \\ \text { ALL } & 27 \% & 69 \% & 80 \% \\ \text { MUST } & 3 \% & 59 \% & 50 \% \\ \text { THINK } & 50 \% & 59 \% & 70 \%\end{array}$


If the pilot data are representative, there are more local implicatures in English than Nausicaa Pouscoulous and Bart Geurts might expect. Overall (see the middle column of Table 5), there were at least $59 \%$ strengthened interpretations in all contexts, including the embedded ones. This supports the intuition that in English, at least, embedded implicatures are computed fairly often. The implicit prosody hypothesis, which predicted more strengthening when some was capitalized than when it was not, wasn't generally supported in the pilot data. ${ }^{1}$ There may well have been implicit prosody effects, but they were not general, and the specific effects suggested by the data will be taken up below in full scale studies (and thus not addressed here).

Before leaving the pilot study, it should be noted that one of the filler items in the pilot was included in order to examine a prediction of the idea that the amount of strengthening of one implicature trigger versus another in any given context may be a function in part of how salient and stable the alternatives to that trigger are. For cardinals, they are highly salient and stable. As children, we learned numbers together with their alternatives by reciting one, two, three, etc. Intuitions suggest that cardinal numbers typically show more strengthening than other implicature triggers. To test the idea that strengthening, computing scalar implicatures, is highly likely with a salient stable scale, the pilot included the items in (16). The idea was that, like cardinals, we learn the letters of the alphabet together with their alternatives, reciting them as $a, b, c$, etc. Thus, if the existence of salient stable alternatives makes the strengthening of an implicature trigger highly likely, we would expect lots of interpretations of (16) as " $\mathrm{G}$ and no farther."

(16) Susie says/can say/CAN say the alphabet to "G".

_ Susie can say the alphabet to "G," but no farther

_ Susie can say the alphabet to "G," and possibly farther

Indeed, 12 out of 15 responses to (16) indicated that participants strengthened (16). This is at least suggestive support for the role of salient stable alternatives in regulating the (baseline) amount of strengthening associated with a particular implicature trigger.

\subsection{Embedding implicatures under has/must}

We turn next to the effect of an embedding verb on pragmatic strengthening processes. HAS/MUST may be assumed to impose a requirement in sentences like

\footnotetext{
${ }^{1}$ The effect of capitalzation may have had an effect throughout the experiment (diminishing s'm). I don't think the capitalization was totally ineffective. For Jenny watched tennis or volleyball $5 / 10$ responses were exclusive, whereas with $O R 5 / 5$ were exclusive, for example.
} 
(17). Consequently it may bias readers to a lower bounded interpretation since requirements usually impose a criterion which must be met and may be exceeded. This bias rather than embedding per se might lead us to expect somewhat fewer strengthened interpretations in (17b) than in (17a). (17d) with explicit focus on HAS/MUST should show substantially fewer strengthened interpretations than (17a-c), on the assumption that it is the bias of the verb rather than embedding per se which influences pragmatic strengthening. This expectation was tested in a written paraphrase selection task, using materials like those in (17).

a. Patrick weeded some of the gardens.

b. Patrick has to weed some of the gardens

c. Patrick has to weed SOME of the gardens.

d. Patrick HAS to weed some of the gardens.

Question: What does Patrick (have to) weed?

1. Some of the gardens but not all of them.

2. Some of the gardens and possibly all of them.

The results are presented in (17'). As expected the presence of has together with emphasis on has (HAS), resulted in a significant drop in strengthening. The mere presence of has resulted in only a small (and not fully significant) drop in strengthening.
a: $84.7 \%$ strengthened
b: $78.1 \%$ strengthened
c: $80.6 \%$ strengthened
d: $66.7 \%$ strengthened

Thus, has may impose a bias toward fewer implicatures when it imposes a requirement.

\subsection{Embedding implicatures under Thinks vs $\underline{\text { Knows }}$}

A written interpretation study compared think and know as the embedding predicates. The assumption that (informational) focus is expected at the end of the sentence would lead us to expect substantial strengthening in $(18 \mathrm{a}, \mathrm{c})$. When thinks/knows is capitalized, presumably alternatives will be activated which may be ordered on a certainty scale, with know at the top of the scale perhaps with is certain/can prove and think presumably lower on the certainty scale but not as low as guess. (Of course other alternatives might also be possible for know - an issue that will be set aside for the present.) Given these assumptions, know may impose a bias for a lower bounded interpretations, like vouch for, because being certain about $\mathrm{x}$ does not imply being certain about something stronger than $\mathrm{x}$. 
(Essentially this explanation was given by one participant in the pilot experiment who received the capitalized KNOW condition.) With respect to thinks/THINKS, several possibilities exist. Perhaps the most interesting is that, because thinks is intermediate on a scale of certainty (from guesses to knows/is certain), perhaps emphasizing thinks will not dramatically change the amount of strengthening, since the perceiver will not know what aspect of thinks is being emphasized - that the speaker is certain to some positive degree, or that the speaker is not certain.

(18) a. Rob thinks Laura saw some of the James Bond movies.

b. Rob THINKS Laura saw some of the James Bond movies.

c. Rob knows Laura saw some of the James Bond movies.

d. Rob KNOWS Laura saw some of the James Bond movies.

Question: That sentence was about Laura seeing

1. some of the James Bond movies but not all of them.

2. some of the James Bond movies and possibly all of them.

(18') Proportion of strengthened readings:
(19a) $0.75 \%$
(19b) $0.74 \%$
(19c) $0.74 \%$
(19d) $\quad 0.66 \%$

The results are summarized in (18'): Sentence (18d) received significantly fewer strengthened interpretations than the other three sentences, which did not differ from each other. Sentences (18a,c and d) behaved as expected. (18b) THINKS behaved like the uncapitalized thinks/knows conditions, perhaps suggesting that its presumed intermediate position on a certainty scale leaves it without a strong bias in our sentences.

\section{Local vs. global computations}

We turn now to a study of the domain widening NPI ever. On the face of it, domain wideners are related to strengthening in two respects. First, domain widening IS a form of strengthening - one that requires even exceptional members of the domain to be taken into account. Second, domain wideners are sensitive to the monotonicity of their contexts, as is strengthening in general. In the study to be reported, ever was placed in a garden path sentence to determine whether the monotonicity properties of a temporary syntactic analysis influence the processing of a sentence. On a view where only global computations of monotonicy are computed, only the monotonicity of the ultimately correct syntactic structure would be expected to matter. On a localist view of implicature 
computation, the monotonicity of the temporary (garden-path) analysis might be expected to exert an influence on the processing of the sentence.

The garden-path structure that will be used is the same as the famous garden-path in (19) (originally due to Thomas Bever). In these structures, The horse raced past the barn is analyzed as a simple main clause until the tensed verb fell is encountered. Then the first syntactic analysis must be reanalyzed to produce a subject phrase containing a reduced relative clause.

The horse raced past the barn fell.

If NPIs such as ever are licensed semantically by DE contexts and monotonicity computations come into play only in terms of the global context of the entire utterance, then a garden-path in the syntactic analysis shouldn't influence the processing of an NPI in the garden-path sentence. The nature of the context, DE for the relative clause or non-DE for the main clause, should be computed with respect to the final syntactic analysis of the sentence. On a local approach, or a syntactic approach, one might expect difficulties if an NPI (a domain widener like ever) appeared to be unlicensed on the first syntactic analysis of the sentence.

In short, strings like $A$ teacher caught... are known to be parsed initially as main clause structures (non-DE). Thus a localist approach, which must keep track of monotonicity during the processing of the sentence, might lead us to expect (20b) to be difficult or odd because the NPI ever will at first appear not to be licensed, i.e., before the syntactic garden-path is reanalyzed. This prediction was tested in a written acceptability judgment study with Charles Clifton, using a 5 point scale. The study tested materials like those in $(21 / 22)$. Examples with disambiguated relative clause structures served as controls $(21 / 22 \mathrm{c}$ and $\mathrm{d})$. A localist view predicts an oddity in the reduced relative clause forms when they contain ever. By contrast, in the full relative clause forms, the processor knows that the structure is a relative clause (DE) before encountering ever. So ever should not appear to be unlicensed in the full relative clause examples.

(20) a. A teacher caught with communist literature won't be allowed to teach Social Studies.

b. A teacher ever caught with communist literature won't be allowed to teach Social Studies.
a. [sing, RRC no ever]
b. [sing, RRC ever]
c. [sing, FRC, no ever]

A man arrested in this country won't break the law here again. A man ever arrested in this country won't break the law here again.

A man who was arrested in this country won't break the law here again. 
d. [sing, FRC, ever]
a. [pl, RRC, no ever]
b. [pl, RRC, ever]
c. [pl, FRC, no ever]
d. [pl, FRC, ever]

A man who was ever arrested in this country won't break the law here again.

Men arrested in this country won't break the law here again.

Men ever arrested in this country won't break the law here again.

Men arrested in this country won't break the law here again.

Men ever arrested in this country won't break the law here again.

The results appear below in terms of mean acceptability $(5=$ good $)$. The Reduced Relative Clause (RRC) was rated worse than the Full Relative Clause (FRC), examples containing ever were rated worse than examples without ever. As predicted there was an interaction of ever and full/reduced RC, with the lowest ratings being the reduced relative ever forms.

Singular: 3.88 - RRC no ever

\subsection{6 - RRC ever \\ 4.00 - FRC no ever \\ 3.62 - FRC ever}

Plurals: 3.68 - RRC no ever

\subsection{4 - RRC ever \\ 3.96 - FRC no ever \\ 3.84 - FRC ever}

The results thus suggest a localist view. Monotonicity properties associated with a temporary syntactic analysis of a sentence influence the processing of a sentence. This is difficult to reconcile with an approach where monotonicity comes into play only in terms of the global utterance.

\section{Summary and Possible Implications}

The main results of the paper may be summarized easily:

- DE contexts give rise to fewer scalar implicatures than their non-DE counterparts

- Effects of DE vs non-DE contexts show up online (in the self-paced reading studies, ever-study) 
- No reliable support was found for fewer implicatures in non-veridical contexts than veridical ones

- Focusing a scalar implicature trigger increases the number of implicatures (presumably by activating the alternatives that form a scale)

- DE effects are not due to a focus confound

- The paucity of embedded implicatures in Pouscoulous and Geurts does not carry over to English

- Effects of epistemic knowledge and focus interact (focusing a propositional attitude verb invokes a scale of certainty, apparently yielding a lower-bounded interpretation for knows; focus appears to interact differently with implicature triggers that are intermediate on their scale and with those that are at or near endpoints).

Overall the results support Chierchia's proposal, though it needn't be implemented in terms of computing and then canceling implicatures in contexts known to be DE. More work must be done to determine whether the effect of DE contexts is to inhibit the drawing of implicatures in the first place, or to cancel implicatures that have been drawn. Similarly strengthening was not completely absent in DE contexts. To a psycholinguist, this is perhaps less surprising than it might be to a semanticist. Sentences have an indefinite number of properties. Participants in a study, even those paying full attention, may not be responding to the properties of interest to the experimenter and participants may be imagining different scenarios from those intended by the experimenter. Thus, studies like those reported here do not necessarily provide an accurate estimate of the absolute number of implicatures drawn. Rather, it is the relation between conditions that may be interpreted securely.

Focus activates alternatives. Differences among implicature triggers are plausibly due to how stable and salient their alternatives are (highly stable for numbers and letters of the alphabet, less stable for some). Both numbers and other triggers yield fewer implicatures in DE contexts than non-DE contexts. Further, the evidence that the monotonicity properties of sentences are computed locally as the parse develops fits well with the idea that some pragmatic processing is automatic, highly structured by the linguistic system, and not strictly global.

\section{References}

Bezuidenhout, Anne and James Cooper Cutting: 2002, 'Literal meaning, minimal propositions, and pragmatic processing', Journal of Pragmatics 34, 433456.

Bott, Lewis and Ira Noveck: 2004, 'Some utterances are underinformative: The onset and time course of scalar inferences', Journal of Memory and Language 51, 437-457. 
Breheny, Richard, Napolean Katsos, and John Williams: 2006, 'Are generalized scalar implicatures generated by default? An on-line investigation into the role of context in generating pragmatic inferences', Cognition 100, 434463.

Carlson, Katy, Michael Walsh Dickey, Lyn Frazier, and Charles Clifton: 2008, 'Information structure expectations in sentence comprehension', Quarterly Journal of Experimental Psychology.

Chierchia, Gennaro: 2004, 'Scalar implicatures, polarity phenomena and the syntax/pragmatics interface'. In A. Belletti (ed.) Structure and Beyond, Oxford University Press.

Chierchia, Gennaro, Lyn Frazier and Charles Clifton: submitted, When basic meanings are (not) enough: Processing scalar implicatures in adult comprehension. University of Massachusetts Occasional Papers in Linguistics 39.

Cutler, Anne and Jerry A. Fodor: 1979, Semantic focus and sentence comprehension. Cognition, 7, 49-59.

Drenhaus, Heiner, Stephen Frisch and Doug Saddy: 2005, Processing negative polarity items: When negation comes through the backdoor. In S. Kepser and Marga Reis (eds.) Linguistic Evidence. Mouton de Gruyter, Berlin.

Frazier, Lyn and Charles Clifton: 1998, Comprehension of sluiced sentences. Language and Cognitive Processes, 13, 499-520.

Frazier, Lyn, Charles Clifton and Florian Schwarz. In progress. Processing scalar implicatures.

Geurts, Bart and Frans Van Der Silk: 2005, 'Monotonicity and processing load', Journal of Semantics 22, 97-117.

Giannakidou, Anatasia: 1998, Polarity Sensitivity as (Non)veridical Dependency. John Benjamins, Amsterdam.

Grice, Herbert Paul: 1989, Studies in the Ways of Words. Harvard, MA:Harvard University Press.

Grodner, Daniel, Natelie Klein, Katie Carbary and Michael K. Tanenhaus: 2008, Experimental evidence for rapid interpretation of pragmatic 'some'. Paper presented at $21^{\text {st }}$ Annual CUNY Conference, March, 2008. Chapel Hill.

Horn, Lawrence: 1972, On the Semantic Properties of Logical Operators in English, Doctoral Dissertation, University of California, Los Angeles.

Horn, Lawrence: 1989, A Natural History of Negation, University of Chicago Press.

Horn, Lawrence: 1992, The said and the unsaid. In C. Barker and D. Dowty (eds.) Proceedings of Semantics and Linguistics Theory II (pp. 163-192) Columbus, OH:Department of Linguistics, Ohio State University.

Hornby, Paul A.: 1974, Surface structure and presupposition. Journal of Verbal Learning and Verbal Behavior, 13, 530-538. 
Huang, Yi Ting and Jesse Snedeker: In progress, From meaning to inference: Evidence for the distinction between lexical semantics and scalar implicature in online processing.

Levinson, Stephen: 2000, Presumptive Meanings, Cambridge,MA: MIT Press.

Noveck, Ira and Andres Posada: 2003, 'Characterizing the time-course of an implicature: an evoked potentials study', Brain and Language 85, 203210.

Noveck, Ira, Raphaele Guelminger, Nicholas Georgieff, and Nelly Labruyere: 2007, What autism can reveal about Every...not Sentences. Journal of Semantics, 24, 73-90.

Panizza, Danielle. and Gennaro Chierchia: In progress, On the role of entailment and scalar implicature in the processing of numbers.

Pouscoulous, Nausicaa and Bart Geurts: 2007, Experimental evidence against local implicatures CSSP Colloquium in Syntax and Semantics in Paris, 2007.

Russell, Benjamin: 2006, Against grammatical computation of scalar implicatures. Journal of Semantics, 23, 361-382.

Sanford, Anthony J. and Patrick Sturt: 2002,Depth of processing in language comprehension: not noticing the evidence. TRENDS in Cognitive Sciences, 6 (9), 382-386.

Sperber, Dan and Deirdre Wilson: 1986, Relevance: Communication and Cognition. Oxford: Blackwell.

Szabolcsi, Anna, Lewis Bott and Brian McElree: Ms., The effect of negative polarity items on inference verification.

\section{Appendix}

\section{A. One time vs. Many time conditionals: Tense}

In a written questionnaire study, 48 participants were asked to choose paraphrases:

(i) a. If John has ten dollars, he'll go to NY for the weekend. Strengthened $55 \%$

b. If John had ten dollars, he went to NY for the weekend. Strengthened $46 \%$

(ii) a. If Sue takes band or a swimming class, she'll be happy. Strengthened $30 \%$

b. If Sue took band or a swimming class, she was happy. Strengthened $29 \%$

There was no significant effect of tense. 


\section{One time vs Many time conditionals: DP-type}

In a one item written interpretation study:

(iii) a. If someone takes art classes or does karate, she'll be happy.

51 exclusive; 61 inclusive

b. If Beth takes art classes or does karate, she'll be happy.

38 exclusive; 72 inclusive

$($ Chi-square $=2.79, \mathrm{p}<.10)$

The studies summarized above suggest there isn't a robust general effect of manytime conditionals behaving more like every than one time conditionals.

\section{B. Pouscoulous and Geurts: Experiments 2 and 3}

Pouscoulous and Geurts' Experiment 2 examined distinct matrix verbs:
a. Jill thinks that Jack read some of the Harry Potter books. $=>$ She thinks he didn't read them all. (Thinks)
b. Jill wants Jack to read some of the Harry Potter books. $=>$ She wants him not to read them all. (Wants)

The results indicated a $65 \%$ endorsement rate for thinks, and a 32\% endorsement rate for wants. The control yielded a $94 \%$ endorsement rate.

They addressed the question of whether the high rate of endorsement for thinks could be evidence for a weakened localist position? They concluded that it could not and note that Russell,2006 shows how a Gricean account can deal with semingly localist inferences in belief sentences. They also argue that the "overall level of positive responses in our first two studies is inflated by the exeprimental paradigm."

Experiment 3 was designed to show that the methodology used in Experiments 1 and 2 inflated the true endorsement rate. To show this presumed inflation, they compared the results of the inference task used above, testing (iii), to results for the same sentence where participants have to decide if (iii) is true of a situation (ii):

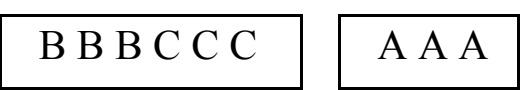

(iii) Some of the B's are in the box on the left. 
Inference in Experiments 1,2:62\% endorse not all

Inference in Experiment. 3: $34 \%$ reject (ii) as applied to ( i)

\section{Domain widening ever}

Several predictions of our account of the oddity of ever in reduced relative clauses have not yet been tested. One prediction is that the effect should disappear when the head of the reduced relative is no man as in (i), since in this case ever should be licensed in either the main clause or the reduced relative syntactic structure.

(i) No man ever arrested in this country will break the law here again.

Another issue concerns the role of the PPI ever. Consider a fully ambiguous sentence like (ii), which can mean "A woman who was once happy with her marriage..." or "A woman, always happy with her marriage,..."

(ii) A woman ever happy with her marriage will find divorce difficult to contemplate.

a. NPI ever: A woman who was ever happy with her marriage...

b. PPI ever: A woman, ever happy with her marriage, ...

The 'generic' property of a relative clause presumably sets up a Downward Entailing (DE) context that licenses the NPI ever (cf. * The police ever arrested $a$ man in this country. but If the police ever arrested a man in this country, he should have been offered a lawyer.). In our reduced relative clause test sentences, ever may at first appear to be unlicensed before the relative clause structure is postulated. Perhaps as a result, the processor considers the possibility that ever is a PPI that widens the domain in positive contexts, yielding an interpretation like that of always. This may exacerbate the difficulty with these examples. 